\title{
Calculation of anisotropic transport coefficients for an ultrarelativistic Boltzmann gas in a magnetic field within a kinetic approach
}

\author{
Zhengyu Chen, ${ }^{1}$ Carsten Greiner, ${ }^{2}$ Anping Huang $\odot,{ }^{1}$ and $\mathrm{Zhe} \mathrm{Xu}^{1, *}$ \\ ${ }^{1}$ Department of Physics, Tsinghua University and Collaborative Innovation Center of Quantum Matter, \\ Beijing 100084, China \\ ${ }^{2}$ Institut für Theoretische Physik, Johann Wolfgang Goethe-Universität Frankfurt, Max-von-Laue-Strasse 1, \\ 60438 Frankfurt am Main, Germany
}

(Received 14 November 2019; accepted 8 March 2020; published 27 March 2020)

\begin{abstract}
According to the Kubo formulas, we employ the $(3+1)$-d parton cascade, Boltzmann approach of multiparton scatterings to calculate the anisotropic transport coefficients (shear viscosity and electric conductivity) for an ultrarelativistic Boltzmann gas in the presence of a magnetic field. The results are compared with those recently obtained by using the Grad's approximation. We find good agreements between both results, which confirms the general use of the derived Kubo formulas for calculating the anisotropic transport coefficients of quark-gluon plasma in a magnetic field.
\end{abstract}

DOI: 10.1103/PhysRevD.101.056020

\section{INTRODUCTION}

The experiments of ultrarelativistic heavy-ion collisions at the Relativistic Heavy Ion Collider and the Large Hadron Collider are believed to reach high enough energies to create the quark-gluon plasma (QGP) [1], which is composed of deconfined quarks and gluons at or close to thermal equilibrium. The QGP behaves like a nearly perfect fluid with a small value of the shear viscosity to the entropy density ratio [2-7]. Because of the existence of a strong magnetic field in the early stage of relativistic heavy-ion collisions [8-11], the QGP may behave differently from the one when ignoring the magnetic field. Actually, the magnetic field breaks the spatial symmetry and leads to the anisotropization of transport coefficients [12-16]. Their values depend on the strength of the magnetic field.

The transport coefficients are important physical quantities characterizing the features of QGP and reflecting the nature of interactions between quarks and gluons. In the last decade, dissipative hydrodynamic models [17-25] have played a very important role in extracting the shear and bulk viscosity of QGP [26] from the flow measurements [4,27-34]. Now it is necessary to develop models based on relativistic magnetohydrodynamics $[35,36]$, in order to study QGP dynamics in the presence of the magnetic field [37-48].

\footnotetext{
*xuzhe@mail.tsinghua.edu.cn
}

Published by the American Physical Society under the terms of the Creative Commons Attribution 4.0 International license. Further distribution of this work must maintain attribution to the author(s) and the published article's title, journal citation, and DOI. Funded by SCOAP.
How large the magnetic effect (also the chiral magnetic effect $[9,38,49,50])$ is depends on the strength of the magnetic field. The magnetic field in the early stage of a noncentral heavy-ion collision stems from the passage of two moving ions and its life time is very short. However, the rapid decrease of this external magnetic field will lead to an electromagnetic induction of QGP, so that the total magnetic field can last longer, if the QGP is a good conductor with a large electric conductivity. Therefore, the value of the electric conductivity of the QGP is essential for the possibility of observing magnetic effects in heavyion collisions.

According to the Green-Kubo relation [51,52], the transport coefficients are related to the correlation functions of the corresponding tensor or flux. In Refs. $[13,15,16]$ Kubo formulas for anisotropic transport coefficients in the presence of a magnetic field are derived with different methods. The calculation of the correlation functions of fluctuating tensor or flux can be realized in kinetic transport models. In this work, we employ the Boltzmann Approach of MultiParton Scattering (BAMPS) [53], which solves the Boltzmann equation for systems of on-shell particles. In the early studies (in the absence of a magnetic field), BAMPS has been used to calculate the shear viscosity of a pQCDbased gluon gas [54,55] and of QGP [56], the electric conductivity of QGP [57], and recently the shear viscosity of ultrarelativistic Boson systems in the presence of a BoseEinstein condensation [58].

For a simple case such as a one-component system with massless Boltzmann particles undergoing isotropic binary elastic collisions, the anisotropic transport coefficients in a magnetic field can be calculated analytically by using Grad's approximations [59]. In this work we consider 
the same particle system as in [59], calculate the anisotropic transport coefficients with BAMPS via the Kubo formulas given in $[13,15,16]$, and compare the results with those obtained in [59]. An agreement of both results will confirm the general use of the derived Kubo formulas for calculating the anisotropic transport coefficients of QGP in a magnetic field.

The paper is organized as follows: In Sec. II we briefly review the equations of magnetohydrodynamics and give the Kubo formulas for the corresponding transport coefficients. In Sec. III we introduce the parton cascade BAMPS and numerical implementations. Subsequently, in Sec. IV we show our numerical results including the influence of the magnetic field on the time evolution of corresponding correlation functions and the values of shear viscosity and electric conductivity coefficients for a onecomponent system of ultrarelativistic Boltzmann particles with isotropic binary scatterings. Finally, we give a conclusion in Sec. V.

We adopt natural units, $\hbar=c=k_{B}=1$. The metric tensor is chosen to be $g^{\mu \nu}=\operatorname{diag}(+,-,-,-)$.

\section{ANISOTROPIC TRANSPORT COEFFICIENTS AND KUBO FORMULAS}

The dynamics of relativistic fluids in an external magnetic field has been studied in Ref. [13]. The authors have found that due to the breaking of the spatial symmetry in the presence of a magnetic field, the dissipative functions contain anisotropic transport coefficients, namely, two bulk viscosity, five shear viscosity, and three electric conductivity coefficients. At first we briefly summarize the results of Ref. [13]. The same results can also be found in Ref. [60].

For a charged particle system, the basic equations of magnetohydrodynamics consist of the conservation laws of energy, momentum, and electric charge, and the constitutive equations for the energy-momentum tensor and the electric current. The conservation laws can be expressed as

$$
\begin{gathered}
\partial_{\mu} j^{\mu}=0, \\
\partial_{\mu} T^{\mu \nu}=F^{\nu \mu} j_{\mu},
\end{gathered}
$$

where $F^{\mu \nu}$ is the electromagnetic field-strength tensor. The electric field is neglected in $[13,15]$ assuming that the electric field is much smaller than the magnetic field, which is a good approximation for QGP produced in heavy-ion collisions for instance. The constitutive equations in the Landau frame read $[13,60]$

$$
\begin{gathered}
j^{\mu}=q n u^{\mu}+\mathcal{J}^{\mu}, \\
T^{\mu \nu}=\varepsilon u^{\mu} u^{\nu}-P_{\perp} \Xi^{\mu \nu}+P_{\|} b^{\mu} b^{\nu}+\mathcal{T}^{\mu \nu},
\end{gathered}
$$

where $u^{\mu}$ is the fluid four-velocity normalized to $u^{2}=1$ and $b^{\mu} \equiv B^{\mu} / B$ with $B^{\mu} \equiv \epsilon^{\mu \nu \alpha \beta} F_{\nu \alpha} u_{\beta} / 2$ and
$B \equiv \sqrt{-B^{\mu} B_{\mu}} \cdot q$ is the particle charge. $\varepsilon$ and $n$ denote the energy and particle number density, respectively. The tensor which projects onto the three-dimensional space orthogonal to the flow velocity $u^{\mu}$ is defined as $\Delta^{\mu \nu} \equiv g^{\mu \nu}-u^{\mu} u^{\nu} . \Xi^{\mu \nu} \equiv \Delta^{\mu \nu}+b^{\mu} b^{\nu}$ is the tensor, which projects onto the two-dimensional space orthogonal to both the flow velocity $u^{\mu}$ and the direction of the magnetic field. $P_{\|}$and $P_{\perp}$ are defined as $P_{\|} \equiv b_{\mu} b_{\nu} T^{\mu \nu}$ and $P_{\perp} \equiv-\Xi_{\mu \nu} T^{\mu \nu} / 2$. The dissipative terms in Eqs. (3) and (4) can be obtained by the derivative expansion to the leading order and have the form in terms of viscosity and electric conductivity coefficients,

$$
\begin{aligned}
& \mathcal{J}^{\mu}=T\left(\kappa_{\perp} \Xi^{\mu \nu} \nabla_{\nu} \alpha-\kappa_{\|} b^{\mu} b^{\nu} \nabla_{\nu} \alpha-\kappa_{\times} b^{\mu \nu} \nabla_{\nu} \alpha\right), \\
& \mathcal{T}^{\mu \nu}= \frac{3}{2} \zeta_{\perp} \Xi^{\mu \nu} \phi+3 \zeta_{\|} b^{\mu} b^{\nu} \psi+2 \eta_{0}\left(w^{\mu \nu}-\frac{1}{3} \Delta^{\mu \nu} \theta\right) \\
&+\eta_{1}\left(\Delta^{\mu \nu}-\frac{3}{2} \Xi^{\mu \nu}\right)\left(\theta-\frac{3}{2} \phi\right) \\
&-2 \eta_{2}\left(b^{\mu} \Xi^{\nu \alpha} b^{\beta}+b^{\nu} \Xi^{\mu \alpha} b^{\beta}\right) w_{\alpha \beta} \\
&-2 \eta_{3}\left(\Xi^{\mu \alpha} b^{\nu \beta}+\Xi^{\nu \alpha} b^{\mu \beta}\right) w_{\alpha \beta} \\
&+2 \eta_{4}\left(b^{\mu \alpha} b^{\nu} b^{\beta}+b^{\nu \alpha} b^{\mu} b^{\beta}\right) w_{\alpha \beta},
\end{aligned}
$$

where $\alpha \equiv \beta \mu \quad\left(\mu\right.$ is the chemical potential), $b^{\mu \nu} \equiv$ $\epsilon^{\mu \nu \alpha \beta} b_{\alpha} u_{\beta}, \quad w^{\mu \nu} \equiv\left(\nabla^{\mu} u^{\nu}+\nabla^{\nu} u^{\mu}\right) / 2, \quad \phi \equiv \Xi^{\mu \nu} w_{\mu \nu}, \quad \psi \equiv$ $b^{\mu} b^{\nu} w_{\mu \nu}, \theta \equiv \partial^{\mu} u_{\mu}$, with $\nabla_{\mu} \equiv \triangle_{\mu \nu} \partial^{\nu}$. The combining coefficients are identified as five shear viscosity $\left(\eta_{0}, \ldots, \eta_{4}\right)$, two bulk viscosity $\left(\zeta_{\perp}, \zeta_{\|}\right)$, and three electric conductivity coefficients $\left(\kappa_{\perp}, \kappa_{\|}, \kappa_{\times}\right)$.

We now summarize Kubo formulas for the anisotropic transport coefficients, which are given in Refs. [13,15,16]. $T^{\mu \nu}$ and $j^{\mu}$ in the formulas given below are taken at the local rest frame. The authors of Ref. [13] used Zubarev's nonequilibrium statistical operator method to relate the anisotropic transport coefficients to correlation functions in equilibrium. The corresponding Kubo formulas are given by $[13,60]$

$$
\begin{gathered}
\eta_{0}=\left.\frac{\partial}{\partial \omega} \operatorname{Im} G_{T^{12} T^{12}}^{R}\right|_{\mathbf{p}=\mathbf{0}, \omega \rightarrow 0}, \\
\eta_{1}=-\frac{4}{3} \eta_{0}-\left.2 \frac{\partial}{\partial \omega} \operatorname{Im} G_{\tilde{P}_{\|} \tilde{P}_{\perp}}^{R}\right|_{\mathbf{p}=\mathbf{0}, \omega \rightarrow 0} \\
\eta_{2}=-\eta_{0}+\left.\frac{\partial}{\partial \omega} \operatorname{Im} G_{T^{13} T^{13}}^{R}\right|_{\mathbf{p}=\mathbf{0}, \omega \rightarrow 0}, \\
\eta_{3}=\left.\frac{1}{4} \frac{\partial}{\partial \omega} \operatorname{Im} G_{\left(T^{11}-T^{22}\right) T^{12}}^{R}\right|_{\mathbf{p}=\mathbf{0}, \omega \rightarrow 0}, \\
\eta_{4}=\left.\frac{\partial}{\partial \omega} \operatorname{Im} G_{T^{13}}^{R} T^{23}\right|_{\mathbf{p}=\mathbf{0}, \omega \rightarrow 0},
\end{gathered}
$$


where the retarded Green's function in quantum statistical theory has the form $G_{A B}^{R} \equiv i \theta\left(x^{0}\right)\langle[A(x), B(0)]\rangle$, and the angular brackets denote the ensemble average in equilibrium. Some other symbols in the above formulas are defined as $\tilde{P}_{\|} \equiv P_{\|}-\Theta_{\beta} \varepsilon-\Theta_{\alpha} n$ and $\tilde{P}_{\perp} \equiv P_{\perp}-\left(\Theta_{\beta}+\right.$ $\left.\Phi_{\beta}\right) \varepsilon-\left(\Theta_{\alpha}+\Phi_{\alpha}\right) n$ with $\Theta_{\beta} \equiv\left(\frac{\partial P}{\partial \varepsilon}\right)_{n, B}, \Phi_{\beta} \equiv-B\left(\frac{\partial M}{\partial \varepsilon}\right)_{n, B}$, $\Theta_{\alpha} \equiv\left(\frac{\partial P}{\partial n}\right)_{\varepsilon, B}$, and $\Phi_{\alpha} \equiv-B\left(\frac{\partial M}{\partial n}\right)_{\varepsilon, B} . M \equiv(\partial P / \partial B)_{T, \mu}$ is the magnetization. More details can be found in $[13,60]$. The coefficients involving magnetization in the definition of $\tilde{P}_{\|}$and $\tilde{P}_{\perp}$ vanish for particles without the dipole moment or spin, which is the case we consider in this work. We also note that a sign mistake in the formula of $\eta_{3}$ occurred in [13] has been corrected.

The Kubo formulas of the viscosity coefficients were also given in Refs. $[15,16]$, where a variational approach and a derivative method were used, respectively. Despite the sign and/or factor differences after unifying the convention for the transport coefficients, the Kubo formulas for the five shear viscosity coefficients are definitely the same among Refs. $[13,15,16]$. Since the bulk viscosity coefficients vanish by considering a massless Boltzmann gas, there is no need to give their Kubo formulas.

The Kubo formulas for the shear viscosity coefficients can be expressed in real space-time with a integration form [61-64]:

$$
\begin{aligned}
& \eta_{0}= \frac{1}{T} \int_{0}^{\infty} d t \int_{V} d^{3} r\left\langle T^{12}(\mathbf{r}, t) T^{12}(0,0)\right\rangle \\
& \eta_{1}=-\frac{4}{3} \eta_{0}-\frac{2}{T} \int_{0}^{\infty} d t \int_{V} d^{3} r\left\langle\tilde{P}_{\|}(\mathbf{r}, t) \tilde{P}_{\perp}(0,0)\right\rangle, \\
& \eta_{2}=-\eta_{0}+\frac{1}{T} \int_{0}^{\infty} d t \int_{V} d^{3} r\left\langle T^{13}(\mathbf{r}, t) T^{13}(0,0)\right\rangle, \\
& \eta_{3}=\frac{1}{4 T} \int_{0}^{\infty} d t \int_{V} d^{3} r \\
& \quad \times\left\langle\left(T^{11}(\mathbf{r}, t)-T^{22}(\mathbf{r}, t)\right) T^{12}(0,0)\right\rangle \\
& \eta_{4}=\frac{1}{T} \int_{0}^{\infty} d t \int_{V} d^{3} r\left\langle T^{13}(\mathbf{r}, t) T^{23}(0,0)\right\rangle
\end{aligned}
$$

where $T$ is the temperature and the angular brackets denote the ensemble average. Without loss of generality, we choose the $z$ direction as the direction of the magnetic field, $B^{\mu}=\left(0,0,0, B_{0}\right)$. Since we consider a homogeneous particle system, the space dependence of the particle flow and the energy-momentum tensor that appeared in the above Kubo formulas can be integrated out directly.

Without the magnetic field $\left(B_{0}=0\right)$, except for $\eta_{0}$, which should be equal to the standard isotropic shear viscosity $\eta$, all other shear viscosity coefficients should vanish. It is obvious for $\eta_{0}, \eta_{2}, \eta_{3}$, and $\eta_{4}$, since $\left\langle T^{13}(\mathbf{r}, t) T^{13}(0,0)\right\rangle$ is equal to $\left\langle T^{12}(\mathbf{r}, t) T^{12}(0,0)\right\rangle$ and both
$\left\langle\left(T^{11}(\mathbf{r}, t)-T^{22}(\mathbf{r}, t)\right) T^{12}(0,0)\right\rangle \quad$ and $\quad\left\langle T^{13}(\mathbf{r}, t) T^{23}(0,0)\right\rangle$ vanish. For the considered system we have $\left\langle\tilde{P}_{\|}(\mathbf{r}\right.$, t) $\left.\tilde{P}_{\perp}(0,0)\right\rangle=-\left\langle\mathcal{T}^{33}(\mathbf{r}, t) \mathcal{T}^{33}(0,0)\right\rangle / 2$ according to the definitions of $\tilde{P}_{\|}$and $\tilde{P}_{\perp}$. From [55,61] we realize that $\quad\left\langle\mathcal{T}^{33}(\mathbf{r}, t) \mathcal{T}^{33}(0,0)\right\rangle=4\left\langle\mathcal{T}^{12}(\mathbf{r}, t) \mathcal{T}^{12}(0,0)\right\rangle / 3=$ $4\left\langle T^{12}(\mathbf{r}, t) T^{12}(0,0)\right\rangle / 3$. Therefore, $\eta_{1}$ vanishes.

Because of the breaking of spatial symmetry by a longitudinal magnetic field, the usual isotropic electric conductivity becomes anisotropic. The transverse conductivity is different from the longitudinal one. Charged particles in the magnetic field experience the Lorentz force, which results in an electric current in the perpendicular direction with respect to both electric and magnetic fields. The electric conductivity associated with this current is called Hall conductivity.

In Ref. [13] the electric conductivity coefficients associated with the diffusion (or heat transfer) are given by the following Kubo formulas:

$$
\begin{aligned}
& \kappa_{\|}=\left.\frac{\partial}{\partial \omega} \operatorname{Im} G_{G^{3} G^{3}}^{R}\right|_{\mathbf{p}=\mathbf{0}, \omega \rightarrow 0}, \\
& \kappa_{\perp}=\left.\frac{\partial}{\partial \omega} \operatorname{Im} G_{G^{1} G^{1}}^{R}\right|_{\mathbf{p}=\mathbf{0}, \omega \rightarrow 0}, \\
& \kappa_{\times}=\left.\frac{\partial}{\partial \omega} \operatorname{Im} G_{G^{1} G^{2}}^{R}\right|_{\mathbf{p}=\mathbf{0}, \omega \rightarrow 0},
\end{aligned}
$$

where $G^{i}(t)=q T^{0 i} / 4 T-j^{i}$ with $i=1,2,3$ denoting the space components. $\kappa_{\|}, \kappa_{\perp}$, and $\kappa_{\times}$are the longitudinal, transverse, and Hall electric conductivity, respectively.

Different from the definitions of $\kappa_{\|}, \kappa_{\perp}$, and $\kappa_{\times}$, in Ref. [16] the electric conductivity (resistivity) coefficients are induced by an electric and a magnetic field, which are perpendicular. The corresponding Kubo formulas are given by

$$
\begin{gathered}
\frac{1}{\omega} \operatorname{Im} G_{j^{3} j^{3}}^{R}(\omega, \mathbf{p}=\mathbf{0})=\sigma_{\|}, \\
\frac{1}{\omega} \operatorname{Im} G_{T^{01} T^{01}}^{R}(\omega, \mathbf{p}=\mathbf{0})=\rho_{\perp} \frac{\omega_{0}^{2}}{B_{0}^{2}} \\
\frac{1}{\omega} \operatorname{Im} G_{T^{01} T^{02}}^{R}(\omega, \mathbf{p}=\mathbf{0})=-\tilde{\rho}_{\perp} \frac{\omega_{0}^{2}}{B_{0}^{2}} \operatorname{sign}\left(B_{0}\right),
\end{gathered}
$$

where $\sigma_{\|}$denotes the longitudinal electric conductivity, and $\rho_{\perp}, \tilde{\rho}_{\perp}$ denote the transverse and Hall electric resistivity, respectively. $\omega_{0}=\varepsilon+P$ is the enthalpy density. All of these formulas are in the limit $\mathbf{p} \rightarrow \mathbf{0}$ and $\omega \rightarrow 0$. We note that $\sigma_{\|}, \rho_{\perp}$, and $\tilde{\rho}_{\perp}$ have no relation to the diffusion (or heat transfer). 
We give now the Kubo formulas in real space-time:

$$
\begin{aligned}
\kappa_{\|}= & \frac{1}{T} \int_{0}^{\infty} d t \int_{V} d^{3} r\left\langle G^{3}(\mathbf{r}, t) G^{3}(0,0)\right\rangle, \\
\kappa_{\perp}= & \frac{1}{T} \int_{0}^{\infty} d t \int_{V} d^{3} r\left\langle G^{1}(\mathbf{r}, t) G^{1}(0,0)\right\rangle, \\
\kappa_{\times}= & \frac{1}{T} \int_{0}^{\infty} d t \int_{V} d^{3} r\left\langle G^{1}(\mathbf{r}, t) G^{2}(0,0)\right\rangle, \\
\sigma_{\|}= & \frac{1}{T} \int_{0}^{\infty} d t \int_{V} d^{3} r\left\langle j^{3}(\mathbf{r}, t) j^{3}(0,0)\right\rangle, \\
\rho_{\perp}= & \frac{B_{0}^{2}}{\omega_{0}^{2} T} \int_{0}^{\infty} d t \int_{V} d^{3} r\left\langle T^{01}(\mathbf{r}, t) T^{01}(0,0)\right\rangle, \\
\tilde{\rho}_{\perp}= & -\operatorname{sign}\left(B_{0}\right) \frac{B_{0}^{2}}{\omega_{0}^{2} T} \int_{0}^{\infty} d t \int_{V} d^{3} r \\
& \times\left\langle T^{01}(\mathbf{r}, t) T^{02}(0,0)\right\rangle .
\end{aligned}
$$

Differences in numerical results of two kinds of electric conductivity coefficients will be shown later in Sec. IV.

Without the magnetic field $\left(B_{0}=0\right)$, the longitudinal and transverse electric conductivity become equal, while the Hall electric conductivity (or resistivity) is meaningless. From the above Kubo formulas, it is obvious that $\kappa_{\|}=\kappa_{\perp}$ and $1 / \rho_{\perp}$ is infinite. We will show in Sec. IV that $\sigma_{\|}$is also infinite.

\section{THE PARTON CASCADE BAMPS AND NUMERICAL IMPLEMENTATIONS}

The time correlation functions in Eqs. (12)-(16) and Eqs. (23)-(28) are evaluated numerically for the considered particle system in a static box with periodic boundary conditions. Initially, particles are distributed homogeneously in coordinate space and thermally in momentum space. The space-time evolution of particles is calculated by employing the parton cascade BAMPS [53]. Coupled to an external electromagnetic field, the Boltzmann equation $[65,66]$ has the form

$$
p^{\mu} \partial_{\mu} f(x, p)+q F^{\mu \nu} p_{\nu} \frac{\partial}{\partial p^{\mu}} f(x, p)=C[f(x, p)],
$$

where $f(x, p)$ is the one-particle phase-space distribution function, and $C[f(x, p)]$ denotes the collision term. Since we restrict ourselves to a single-component gas of particles carrying no dipole moment or spin, the system will have vanishing magnetization and polarization and the magnetic field $F^{\mu \nu}$ involves only a Lorentz force. In the presence of electromagnetic fields, the local equilibrium distribution function for Boltzmann particles has the following general form [66]:

$$
f^{e q}(x, p)=\exp \left[-\frac{\left(p^{\mu}+q A^{\mu}\right) u_{\mu}-\mu}{T}\right]
$$

where $A^{\mu}$ is the four-vector potential corresponding to electromagnetic fields and $\mu$ is the chemical potential. Since we neglect the electric field, in the local rest frame, the particle distribution in equilibrium has the form:

$$
f^{e q}(x, p)=\exp \left(-\frac{E-\mu}{T}\right)
$$

where $E$ is the particle energy. For massless particles, the chemical potential $\mu=0$.

The microscopic interaction processes among particles are simulated via Monte Carlo techniques based on the stochastic interpretation of transition rates [53]. In order to improve the numerical accuracy, the test particle method [53] is introduced. The particle number is artificially increased by a factor of $N_{\text {test }}$, while the interaction cross section is reduced by the same factor simultaneously. Thus, the physical evolution of the particle system is not influenced by this implementation. The collision probability for binary elastic scattering in a spatial cell of a volume of $\Delta V$ and within a time step $\Delta t$ is

$$
P_{22}=v_{\text {rel }} \frac{\sigma_{22}}{N_{\text {test }}} \frac{\Delta t}{\Delta V}
$$

$v_{\text {rel }}=s /\left(2 E_{1} E_{2}\right)$ is the relative velocity of the incoming particles with energy $E_{1}$ and $E_{2}, s$ is the invariant mass, and $\sigma_{22}$ is the total cross section of elastic binary scatterings. We consider the magnetic field $\vec{B}$ to be constant and homogeneous, pointing in the $z$ direction. Thus, the Lorentz force, $\vec{F}_{L}=q \vec{v} \times \vec{B}$, will change the directions (while not the magnitude) of particles' transverse momenta for every computational time step $\Delta t$. Between the collisions, the particles will move in a circle in the transverse plane, while they propagate via free streaming in the $z$ direction.

According to the physical definition, the electric current $j^{\mu}$ and energy-momentum tensor $T^{\mu \nu}$ are calculated as

$$
\begin{gathered}
j^{\mu}(t)=\frac{q}{V N_{\text {test }}} \sum_{i=1}^{N} \frac{p_{i}^{\mu}}{E_{i}}, \\
T^{\mu \nu}(t)=\frac{1}{V N_{\text {test }}} \sum_{i=1}^{N} \frac{p_{i}^{\mu} p_{i}^{\nu}}{E_{i}},
\end{gathered}
$$

where the sum is running over all the $N$ test particles in the box at time $t$ and $V$ is the volume of the box. The correlation is calculated at discrete and equally distributed time steps $t_{l}=t_{0}, t_{1} \ldots, t_{K}$ by time average in the limit $t_{K} \rightarrow \infty$, 

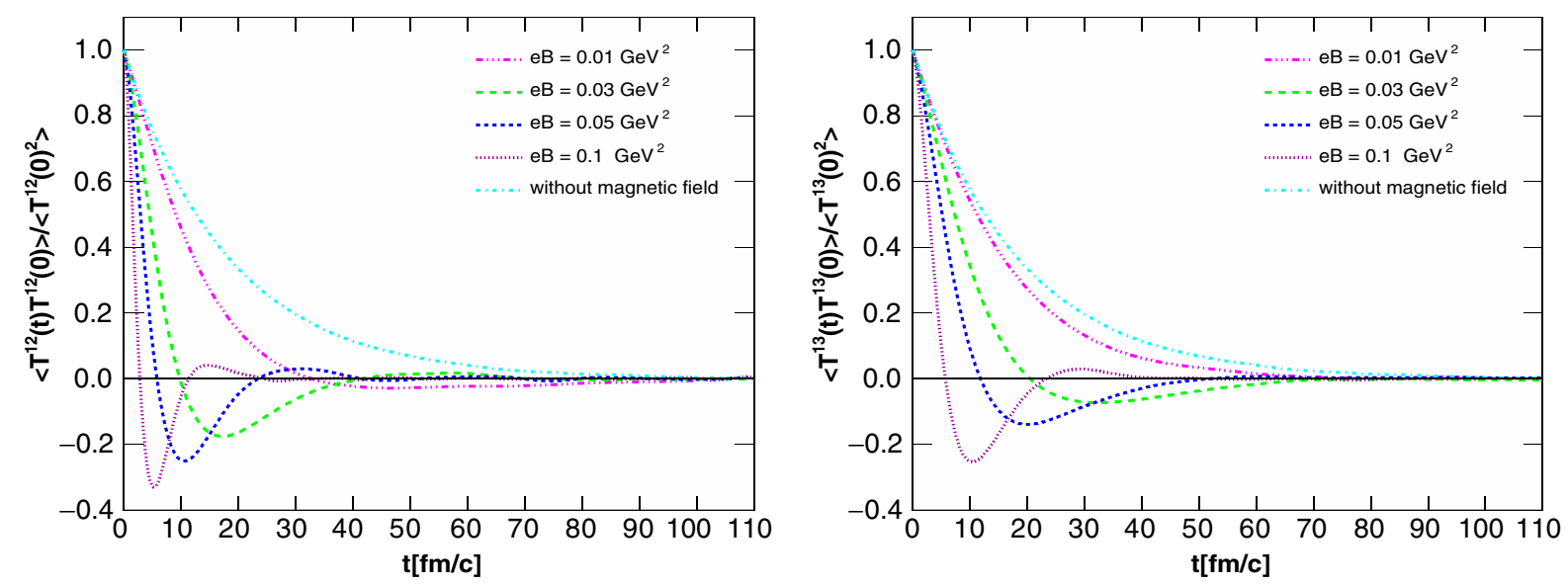

FIG. 1. Time evolution of $\left\langle T^{12}(t) T^{12}(0)\right\rangle$ and $\left\langle T^{13}(t) T^{13}(0)\right\rangle$ with various magnetic field strengths. The results are normalized by their initial values.

$$
C\left(t_{l}\right)=\frac{1}{s_{\max }} \sum_{s=0}^{s_{\max }} A\left(t_{s}\right) B\left(t_{s}+t_{l}\right), \quad s_{\max }=K-l .
$$

$A(t)$ and $B(t)$ represent the component of the particle flow or energy-momentum tensor. The ensemble average is realized by $N_{\text {run }}$ individual initialization. In the presence of a magnetic field, some of the correlation functions oscillate instead of an exponential decrease (as we will show in the next section). Therefore, we have to evolve the systems to a much longer time until the correlations become small.

\section{NUMERICAL RESULTS}

We consider a system of massless Boltzmann particles with a positive charge $e$. Initially particles are sampled in momentum space according to the Boltzmann distribution $f(x, p)=e^{-E / T}$ with a temperature of $T=400 \mathrm{MeV}$. Since the magnetic fields in noncentral $\mathrm{Au}-\mathrm{Au}$ collisions at the Relativistic Heavy Ion Collider can reach $e B \sim m_{\pi}^{2} \sim$ $0.02 \mathrm{GeV}^{2}[67]$ and those at the Large Hadron Collider are 10 times larger [8], we choose in this work $e B=$ $0,0.01,0.03,0.05,0.1 \mathrm{GeV}^{2}$. The chosen magnitude of the magnetic fields is in the region $\sqrt{e B}<T$, where the Landau quantization of the particles' cyclotron motion can be neglected [59]. We assume that particles carry no dipole moment or spin, so that the gas has vanishing magnetization and polarization. Since the Lorentz force does not change the magnitude of the momentum and the isotropy of the momentum distribution, the system will always stay in equilibrium. We consider binary elastic collisions only. The total cross section is set to be a constant value $\left(\sigma_{22}=1 \mathrm{mb}\right)$, and the particles scatter isotropically.

In the following we show the results of shear viscosity and electric conductivity coefficients in the presence of a magnetic field, respectively.

\section{A. Shear viscosity coefficients}

The time evolution of correlation functions $\left\langle T^{12}(t) T^{12}(0)\right\rangle$ and $\left\langle T^{13}(t) T^{13}(0)\right\rangle$, which determine the shear viscosity coefficients $\eta_{0}$ and $\eta_{2}$, are shown in Fig. 1. We can see that the correlation functions behave quite differently from those without magnetic field. The correlation functions no longer decrease exponentially. The presence of the magnetic field induces the oscillations of the correlation function because the Lorentz force changes the sign of $T^{12}$ and $T^{13}$. For a single particle under cyclotron motion, the absolute value of $p_{x} p_{y}$ does not change after $1 / 4,1 / 2,3 / 4$, and 1 period, when compared with its initial absolute value. The sign of $p_{x} p_{y}$ changes after $1 / 4$ and $3 / 4$ period, while it does not change after $1 / 2$ and 1 period. The period of a cyclotron motion is proportional to the magnitude of momentum. After sum over all particles and average over all runs, the time evolution of $\left\langle T^{12}(t) T^{12}(0)\right\rangle$ shows the oscillation behavior with peaks and valleys. Compared with $\left\langle T^{12}(t) T^{12}(0)\right\rangle$, the oscillation frequencies of $\left\langle T^{13}(t) T^{13}(0)\right\rangle$ are smaller, since the

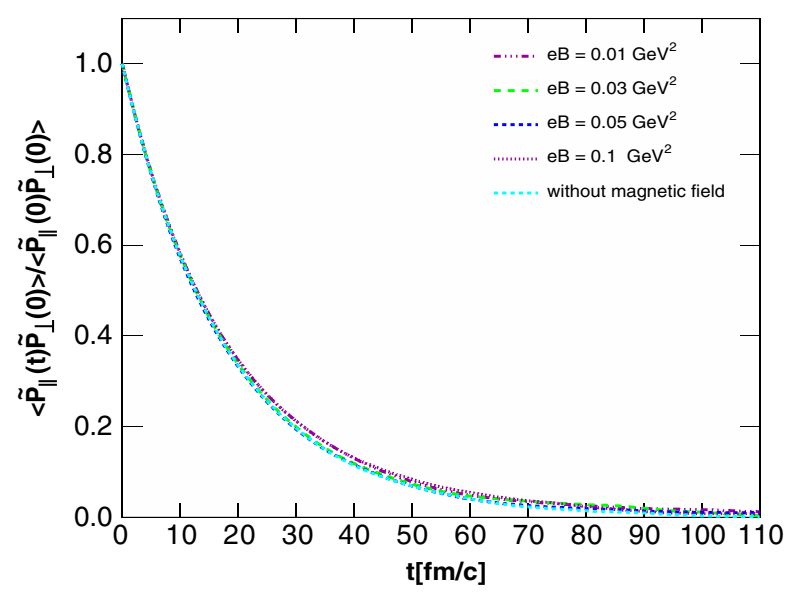

FIG. 2. Same as Fig. 1, but for $\left\langle\tilde{P}_{\|}(t) \tilde{P}_{\perp}(0)\right\rangle$. 

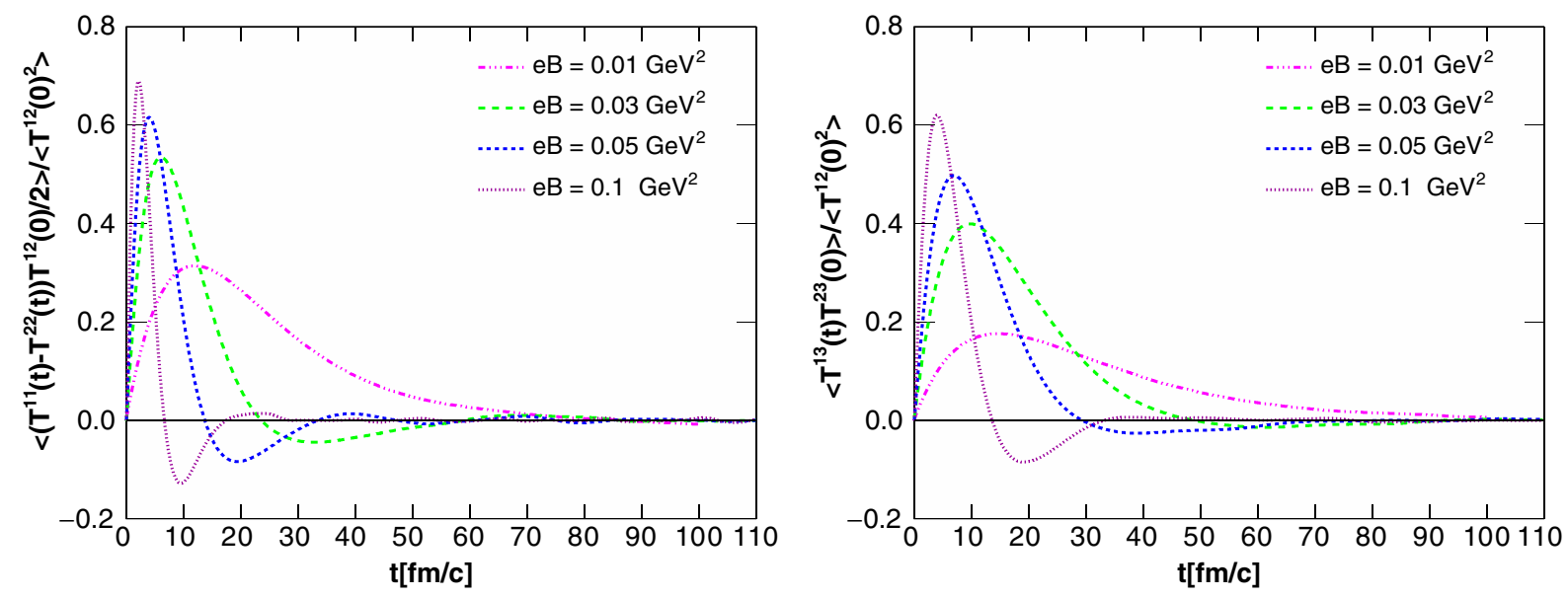

FIG. 3. Time evolution of $\left\langle\left(T^{11}(t)-T^{22}(t)\right) T^{12}(0)\right\rangle / 2$ and $\left\langle\left(T^{13}(t)\right) T^{23}(0)\right\rangle$ with various magnetic field strengths. The results are normalized by $\left\langle\left(T^{12}(0)\right)^{2}\right\rangle$. The results without the magnetic field are zero.

momenta in the $z$ direction are not influenced by the Lorentz force and the value of $p_{x} p_{z}$ will change its sign after $1 / 2$ period, when compared with its initial value.

The correlation function $\left\langle\tilde{P}_{\|}(t) \tilde{P}_{\perp}(0)\right\rangle$, which determines the shear viscosity coefficient $\eta_{1}$, is shown in Fig. 2. For the system we have considered in this work, we have $\tilde{P}_{\|}=T^{33}-T^{00} / 3$ and $\tilde{P}_{\perp}=\left(T^{11}+T^{22}\right) / 2-T^{00} / 3=$ $T^{00} / 6-T^{33} / 2$. From Fig. 2 we see that the magnetic field has no influence on this correlation function despite the small deviation due to numerical fluctuations. This is because the correlation function only involves particles' energy and momentum in the $z$ direction, and both of them are not affected by the magnetic field.

It is obvious that the correlation functions $\left\langle\left(T^{11}(t)-\right.\right.$ $\left.\left.T^{22}(t)\right) T^{12}(0)\right\rangle$ and $\left\langle\left(T^{13}(t)\right) T^{23}(0)\right\rangle$ will vanish if there is no magnetic field. With the magnetic field, the two correlation functions, corresponding to $\eta_{3}$ and $\eta_{4}$, respectively, oscillate due to the same reason for $\left\langle T^{12}(t) T^{12}(0)\right\rangle$ and $\left\langle T^{13}(t) T^{13}(0)\right\rangle$, as seen in Fig. 3. The sign of $p_{x} p_{x}-$ $p_{y} p_{y}$ changes after $1 / 4$ and $3 / 4$ period and is unchanged after $1 / 2$ and 1 period, when compared with its initial value. The two correlation functions behave similarly. The stronger the magnitude of the magnetic field, the earlier the maximum value is reached and the larger is the value.

Figure 4 shows the five anisotropic shear viscosity coefficients for various values of $\xi_{B}=\lambda_{m f p} / R_{T}$, where the mean free path $\lambda_{m f p}=1 /\left(n \sigma_{22}\right)$ is fixed and $R_{T}=$ $T /(e B)$ varies by varying $e B . R_{T}$ denotes the Larmor radius of a particle with the transverse momentum being equal to the temperature. The five shear viscosity coefficients are normalized by the standard isotropic shear viscosity $\eta=$ $4 \lambda_{m f p} P / 3$ obtained without the magnetic field. We see that except for $\eta_{1}$, which is almost constant at the large magnetic field, all the other shear viscosity coefficients are decreasing.
The five anisotropic shear viscosity coefficients of a massless Boltzmann gas undergoing binary isotropic elastic collisions in a magnetic field have been derived analytically in Ref. [59] by using the method of 14-moment Grad's approximation. We list the results below:

$$
\begin{aligned}
\eta_{0} & =\frac{12 \lambda_{m f p} P}{9+4 \xi_{B}^{2}}, \quad \eta_{1}=\frac{64}{9} \frac{\xi_{B}^{2} \lambda_{m f p} P}{9+4 \xi_{B}^{2}}, \\
\eta_{2} & =\frac{36 \xi_{B}^{2} \lambda_{m f p} P}{\left[9+4 \xi_{B}^{2}\right]\left[9+\xi_{B}^{2}\right]}, \quad \eta_{3}=\frac{4 \xi_{B} \lambda_{m f p} P}{9+4 \xi_{B}^{2}}, \\
\eta_{4} & =\frac{4 \xi_{B} \lambda_{m f p} P}{9+\xi_{B}^{2}} .
\end{aligned}
$$

The analytical results are depicted by different curves in Fig. 4. We see excellent agreements with our numerical results. From the formulas in Eq. (36) it is clear that $\eta_{1} / \eta$

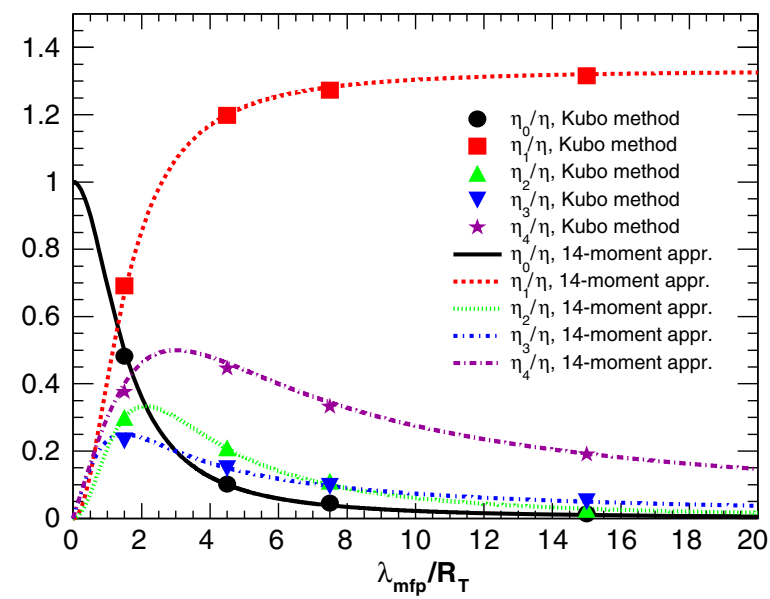

FIG. 4. The magnetic field dependence of the shear viscosity coefficients scaled by the standard isotropic shear viscosity $\eta$. The analytical results from Eq. (36) are shown by the curves for comparisons. 


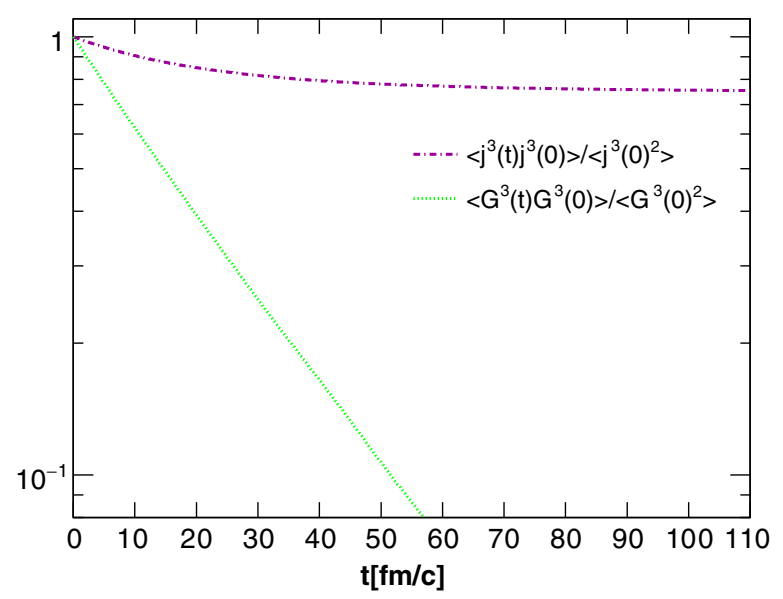

FIG. 5. Time evolution of $\left\langle G^{3}(t) G^{3}(0)\right\rangle$ and $\left\langle j^{3}(t) j^{3}(0)\right\rangle$. The results are normalized by their initial values.

goes to a constant of $4 / 3$ for large $\xi_{B}$ (large $e B$ ), while all the other shear viscosity coefficients go to zero.

Furthermore, by equating Eqs. (12) and (14) to $\eta_{0}$ and $\eta_{2}$ in Eq. (36) we find that

$$
\begin{aligned}
& \frac{V}{T} \int_{0}^{\infty} d t\left\langle T^{12}(\mathbf{r}, t) T^{12}(0,0)\right\rangle=\frac{12 \lambda_{m f p} P}{9+4 \xi_{B}^{2}}, \\
& \frac{V}{T} \int_{0}^{\infty} d t\left\langle T^{13}(\mathbf{r}, t) T^{13}(0,0)\right\rangle=\frac{12 \lambda_{m f p} P}{9+\xi_{B}^{2}} .
\end{aligned}
$$

Therefore, the correlation function $\left\langle T^{12}(t) T^{12}(0)\right\rangle$ at $\xi_{B}$ (or $B$ ) is the same as $\left\langle T^{13}(t) T^{13}(0)\right\rangle$ at $2 \xi_{B}$ (or $2 B$ ). This behavior can be observed in Fig. 1. A similar scaling behavior between $\left\langle\left(T^{11}(t)-T^{22}(t)\right) T^{12}(0)\right\rangle / 2$ and $\left\langle\left(T^{13}(t)\right) T^{23}(0)\right\rangle$ seen in Fig. 3 can also be explained by equating Eqs. (15) and (16) to $\eta_{3}$ and $\eta_{4}$ in Eq. (36).

\section{B. Electric conductivity coefficients}

First, we calculate the longitudinal electric conductivity within BAMPS by applying the Kubo formulas, Eq. (23) and Eq. (26), respectively. $\sigma_{\|}$is induced purely by an electric field, while $\kappa_{\|}$is related to the diffusion (or heat transfer). The correlation functions that determine $\kappa_{\|}$and $\sigma_{\|}$ are $\left\langle G^{3}(t) G^{3}(0)\right\rangle$ and $\left\langle j^{3}(t) j^{3}(0)\right\rangle$. We note that these two correlation functions (also $\kappa_{\|}$and $\sigma_{\|}$) are not influenced by the magnetic field, since the Lorentz force does not affect the dynamics in the direction of the magnetic field. In Fig. 5 we show the time evolution of the two correlation functions and see that $\left\langle G^{3}(t) G^{3}(0)\right\rangle$ decreases to zero, while $\left\langle j^{3}(t) j^{3}(0)\right\rangle$ approaches a nonzero value. The latter indicates an infinite large electric conductivity $\sigma_{\|}$, which is true for a one-component system of charged particles, since there is no energy loss of changed particles in each collision. We mention that the electric conductivity of a multicomponent system such as the quark-gluon plasma has been calculated in [57] within BAMPS without a magnetic field.

The electric conductivity $\kappa_{\|}$, which is related to the diffusion (or heat transfer), is finite. In the top panel of Fig. $8, \kappa_{\|}$scaled by the temperature $T$ are shown by the solid symbols. Charges are multiplied out in the results with $e^{2}=4 \pi / 137$. The electric conductivity calculated here is related with the diffusion coefficient by the WiedemannFranz law. In Ref. [68] the diffusion coefficients for a onecomponent system without a magnetic field are calculated by using the 14-, 23-, 32-, and 41-moment Grad's method. To make comparisons, the results obtained in [68] are multiplied by $e^{2} / T$ according to the Wiedemann-Franz law and shown in the top panel of Fig. 8 by the dashed and dotted lines corresponding to the 14- and 41-moment approximation. We see that our numerical results are about $17 \%$ smaller than those in the 14-moment approximation, while they agree nicely with those in the 41-moment approximation.
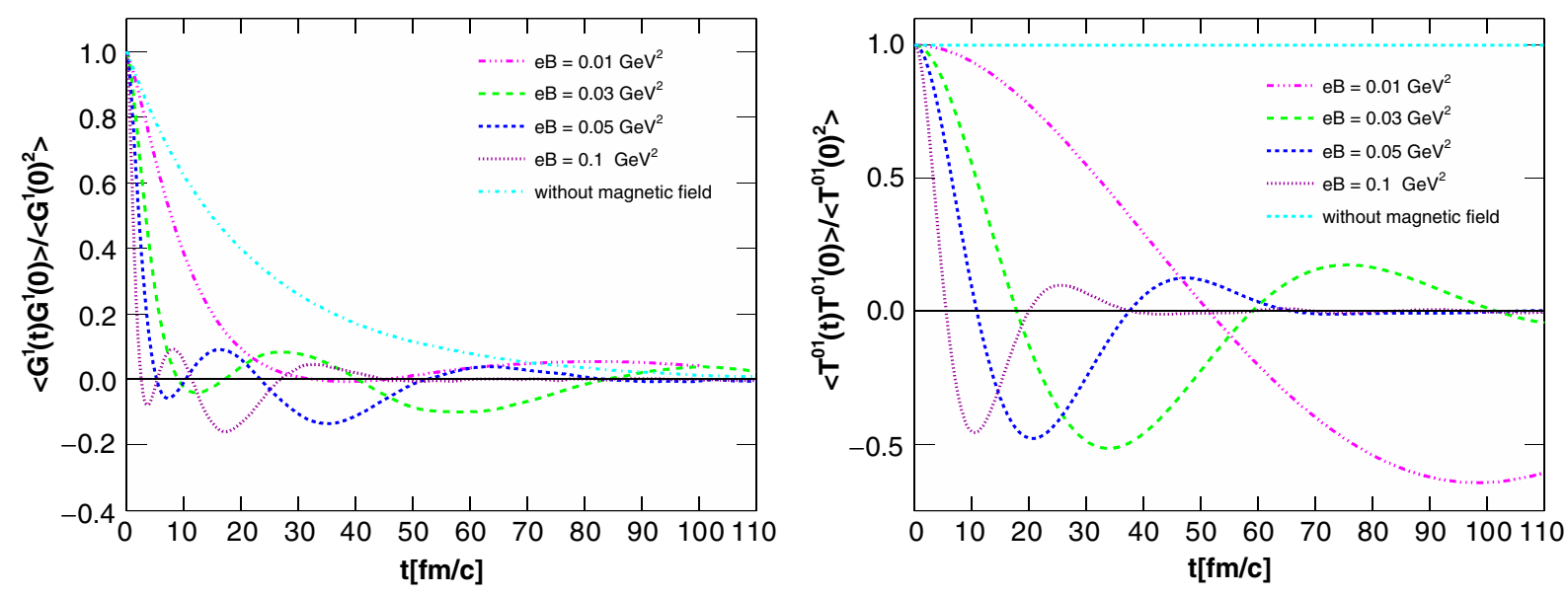

FIG. 6. Same as Fig. 1, but for $\left\langle G^{1}(t) G^{1}(0)\right\rangle$ and $\left\langle T^{01}(t) T^{01}(0)\right\rangle$. 

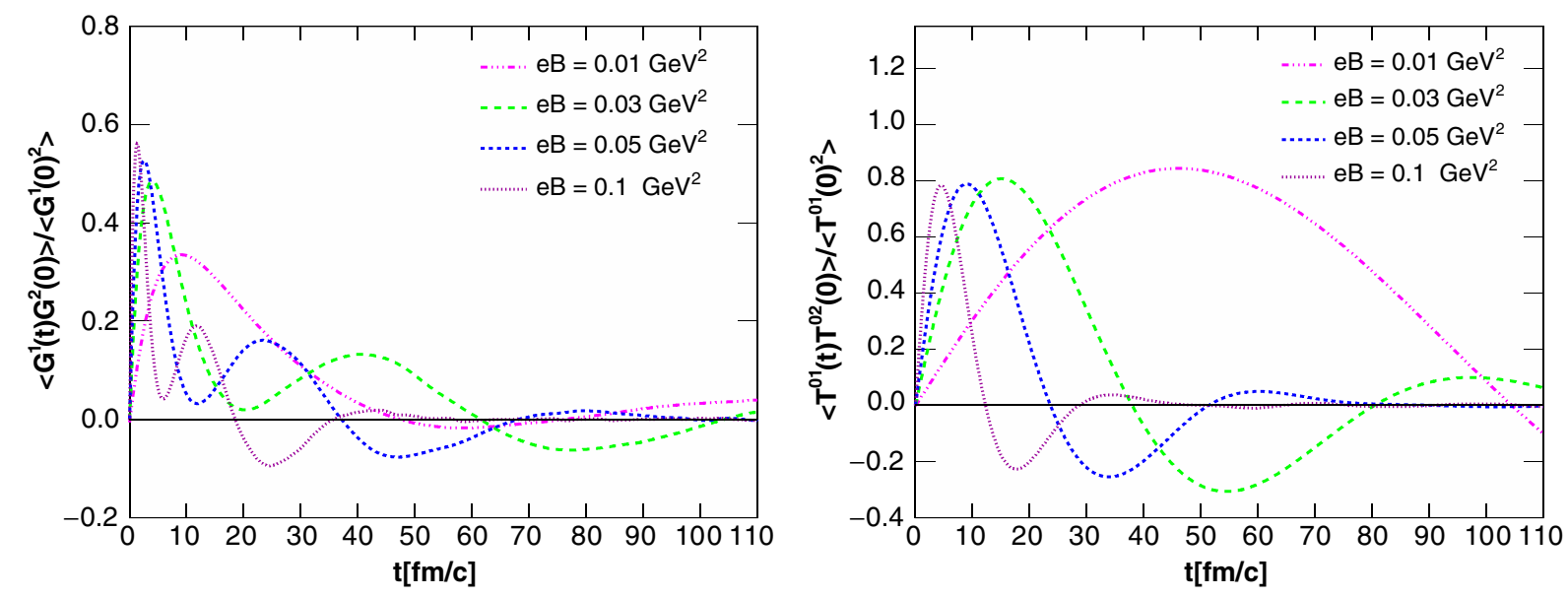

FIG. 7. Time evolution of $\left\langle G^{1}(t) G^{2}(0)\right\rangle$ and $\left\langle T^{01}(t) T^{02}(0)\right\rangle$ with various magnetic field strengths. The results are normalized by $\left\langle\left(G^{1}(0)\right)^{2}\right\rangle$ and $\left\langle\left(T^{01}(0)\right)^{2}\right\rangle$, respectively. The results without the magnetic field are zero.

We now turn to calculate the transverse and Hall electric conductivity. The time evolution of the correlation functions corresponding to these conductivity coefficients [see

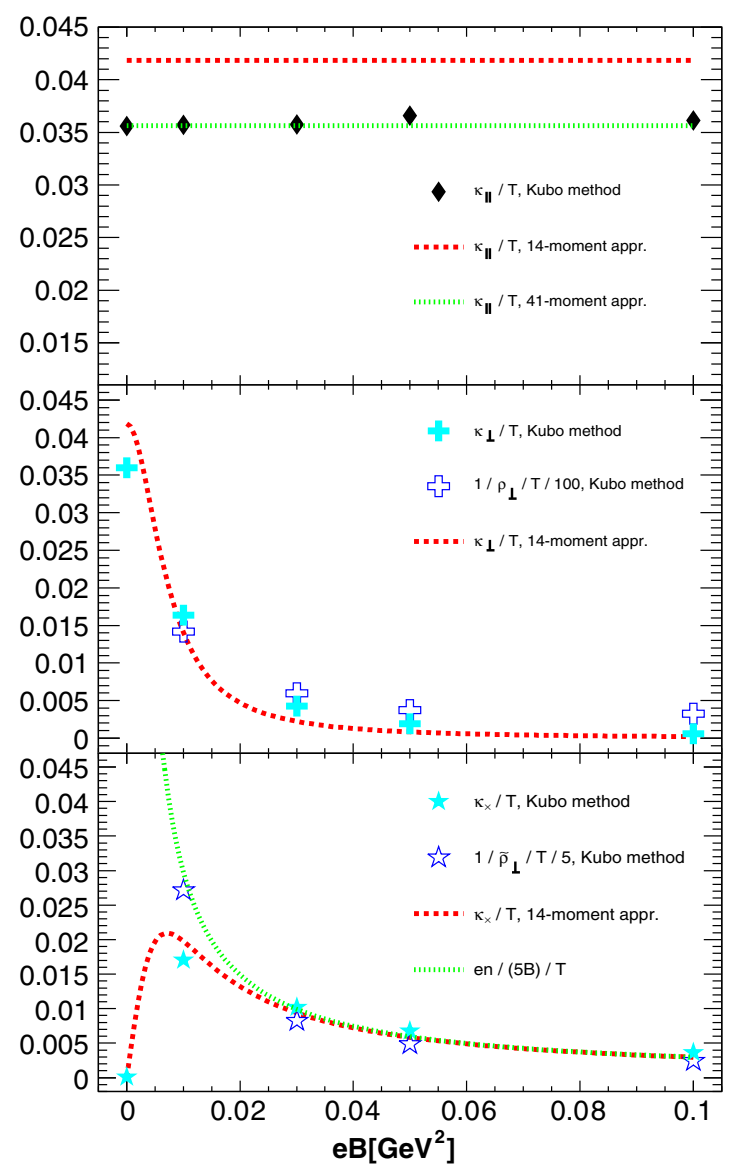

FIG. 8. The magnetic field dependence of the electric conductivity coefficients scaled by the temperature. The analytical results from the 14-moment approximation [see Eq. (39)] and from the 41-moment approximation [68] are shown by the curves for comparisons.
Eqs. (24), (25), (27), and (28)] are shown in Figs. 6 and 7. As explained for Figs. 1 and 3, the oscillation of the correlation functions comes from the cyclotron motion of particles under the Lorentz force. The transverse conductivity coefficients $\kappa_{\perp}$ and $1 / \rho_{\perp}$ scaled by $T$ are depicted in the middle panel of Fig. 8 by the solid and open symbols, respectively. The values of $1 / \rho_{\perp}$ are divided by a factor of 100. At $B=0,1 / \rho_{\perp}$ is infinite [see Eq. (27)], while $\kappa_{\perp}$ is finite. Both are equal to the longitudinal electric conductivity $\sigma_{\|}$and $\kappa_{\|}$, respectively. From the middle panel of Fig. 8 we also see that both $\kappa_{\perp}$ and $1 / \rho_{\perp}$ become smaller for stronger magnetic field strength and $1 / \rho_{\perp}$ is roughly 100 times larger than $\kappa_{\perp}$.

In the bottom panel of Fig. 8 we show the Hall electric conductivity $\kappa_{\times}$and $1 / \tilde{\rho}_{\perp}$ scaled by $T$. The latter is divided by a factor of 5 for comparisons. At $B=0,1 / \tilde{\rho}_{\perp}$ is infinite [see Eq. (28)], while $\kappa_{\times}$is zero. $1 / \tilde{\rho}_{\perp}$ agrees with the classical result $e n / B$ when comparing with the dotted curve in the bottom panel of Fig. 8. (Remember that $1 / \tilde{\rho}_{\perp}$ has been divided by a factor of 5.) With increasing $B, \kappa_{\times}$ increases first and then decreases as $e n /(5 B)$. Thus, $1 / \tilde{\rho}_{\perp}$ is almost 5 times larger than $\kappa_{\times}$. We realize that the electric conductivity coefficients induced by an electric field are always larger than those related with the diffusion (or heat transfer).

The anisotropic diffusion coefficients of a onecomponent system in a magnetic field have been calculated in Ref. [59] by using the 14-moment Grad's method. We multiply these results by $e^{2} / T$ to obtain the electric conductivity coefficients according to the WiedemannFranz law [69]:

$$
\begin{aligned}
\kappa_{\|} & =\frac{3 e^{2} \lambda_{m f p} n}{16 T}, \quad \kappa_{\perp}=\frac{48 e^{2} \lambda_{m f p} n}{\left(256+225 \xi_{B}^{2}\right) T}, \\
\kappa_{\times} & =\frac{45 e^{2} \xi_{B} \lambda_{m f p} n}{\left(256+225 \xi_{B}^{2}\right) T} .
\end{aligned}
$$


$\kappa_{\|}$is exactly the same as that obtained from [68] and has been shown in the top panel of Fig. 8. $\kappa_{\perp}$ and $\kappa_{\times}$from Eq. (39) are depicted by the dashed curves (scaled by $T$ ) in the middle and bottom panels of Fig. 8. We see agreements with the numerical results.

\section{CONCLUSIONS}

In this work, we have calculated the anisotropic transport coefficients of relativistic fluids in the presence of a magnetic field according to the Kubo formulas given in Refs. $[13,16]$. The time correlations of the components of the energy-momentum tensor and electric current, which are fluctuating in time at thermal equilibrium, are calculated numerically within the kinetic transport approach BAMPS. For comparisons with results from the early studies we have considered a massless one-component Boltzmann gas with isotropic binary collisions, although calculations within BAMPS can be performed for multicomponent systems with more complicated scattering processes such like $\mathrm{pQCD}$ (in)elastic scatterings of gluons and quarks [56].

We have found that the magnetic field dependence of the five shear viscosity coefficients that we achieved agrees perfectly with the analytical results obtained by using the 14-moment Grad's approximations [59]. For strong magnetic field, $\eta_{1}$ approaches 4/3-fold of $\eta$ (the standard shear viscosity without the magnetic field) and all the other shear viscosity coefficients decrease to zero.

We have also compared two kinds of electric conductivity coefficients with each other. One electric conductivity coefficients are associated with the diffusion (or heat transfer), another coefficients are induced by an electric field and have no cross effect with the diffusion constant (or heat conductivity). We found that the three electric conductivity coefficients associated with the diffusion are always smaller than those induced by an electric field. In addition, the magnetic field dependence of the three electric conductivity coefficients associated with the diffusion agrees well with the results from the 14-moment Grad's approximations [59]. A better agreement for the longitudinal electric conductivity is seen when comparing the result from the 41-moment approximation [68].

The agreements between the numerical and analytical results on the anisotropic transport coefficients for a onecomponent system of Boltzmann particles with isotropic scatterings confirm the general use of the derived Kubo formulas for multicomponent particle systems with more complicated scattering processes. Calculations of the anisotropic transport coefficients for a multicomponent system in a strong magnetic field such like the QGP produced in heavy-ion collisions are in progress. New results will be shown in a future publication.

\section{ACKNOWLEDGMENTS}

The authors would like to thank Xu-Guang Huang and Dirk H. Rischke for fruitful discussions. This work was financially supported by the National Natural Science Foundation of China under Grants No. 11575092, No. 11890712, and No. 11775123, and the Major State Basic Research Development Program in China under Grant No. 2015CB856903. C. G. acknowledges support by the Deutsche Forschungsgemeinschaft (DFG) through the Grant No. CRC-TR 211 "Strong-interaction matter under extreme conditions." The BAMPS simulations were performed at Tsinghua National Laboratory for Information Science and Technology and on TianHe-1(A) at the National Supercomputer Center in Tianjin.
[1] C. Gale, S. Jeon, and B. Schenke, Int. J. Mod. Phys. A 28, 1340011 (2013).

[2] J. Adams et al. (STAR Collaboration), Nucl. Phys. A757, 102 (2005).

[3] K. Adcox et al. (PHENIX Collaboration), Nucl. Phys. A757, 184 (2005).

[4] K. Aamodt et al. (ALICE Collaboration), Phys. Rev. Lett. 105, 252302 (2010).

[5] M. Gyulassy and L. McLerran, Nucl. Phys. A750, 30 (2005).

[6] I. Arsene et al. (BRAHMS Collaboration), Nucl. Phys. A757, 1 (2005).

[7] B. B. Back et al., Nucl. Phys. A757, 28 (2005).

[8] V. Skokov, A. Y. Illarionov, and V. Toneev, Int. J. Mod. Phys. A 24, 5925 (2009).

[9] W. T. Deng and X. G. Huang, Phys. Rev. C 85, 044907 (2012).
[10] K. Tuchin, Phys. Rev. C 88, 024911 (2013).

[11] A. Bzdak and V. Skokov, Phys. Lett. B 710, 171 (2012).

[12] X. G. Huang, M. Huang, D. H. Rischke, and A. Sedrakian, Phys. Rev. D 81, 045015 (2010).

[13] X. G. Huang, A. Sedrakian, and D. H. Rischke, Ann. Phys. (Amsterdam) 326, 3075 (2011).

[14] R. Critelli, S. I. Finazzo, M. Zaniboni, and J. Noronha, Phys. Rev. D 90, 066006 (2014).

[15] S. I. Finazzo, R. Critelli, R. Rougemont, and J. Noronha, Phys. Rev. D 94, 054020 (2016); 96, 019903 (E) (2017).

[16] J. Hernandez and P. Kovtun, J. High Energy Phys. 05 (2017) 001.

[17] C. Shen, S. A. Bass, T. Hirano, P. Huovinen, Z. Qiu, H. Song, and U. Heinz, J. Phys. G 38, 124045 (2011). 
[18] P. Romatschke and U. Romatschke, Phys. Rev. Lett. 99, 172301 (2007); M. Luzum and P. Romatschke, Phys. Rev. C 78, 034915 (2008); 79, 039903(E) (2009).

[19] H. Song and U. W. Heinz, Phys. Lett. B 658, 279 (2008); Phys. Rev. C 78, 024902 (2008).

[20] P. Bozek and I. Wyskiel-Piekarska, Phys. Rev. C 85, 064915 (2012).

[21] V. Roy, A. K. Chaudhuri, and B. Mohanty, Phys. Rev. C 86, 014902 (2012).

[22] H. Niemi, G. S. Denicol, P. Huovinen, E. Molnar, and D. H. Rischke, Phys. Rev. C 86, 014909 (2012).

[23] U. Heinz, C. Shen, and H. Song, AIP Conf. Proc. 1441, 766 (2012).

[24] B. Schenke, S. Jeon, and C. Gale, Phys. Rev. C 85, 024901 (2012).

[25] P. Romatschke and U. Romatschke, arXiv:1712.05815.

[26] N. Astrakhantsev, V. Braguta, and A. Kotov, J. High Energy Phys. 04 (2017) 101; Phys. Rev. D 98, 054515 (2018).

[27] K. H. Ackermann et al. (STAR Collaboration), Phys. Rev. Lett. 86, 402 (2001).

[28] S. S. Adler et al. (PHENIX Collaboration), Phys. Rev. Lett. 91, 182301 (2003).

[29] J. Adams et al. (STAR Collaboration), Phys. Rev. Lett. 92, 052302 (2004).

[30] B. B. Back et al. (PHOBOS Collaboration), Phys. Rev. Lett. 94, 122303 (2005).

[31] B. Alver et al. (PHOBOS Collaboration), Phys. Rev. Lett. 98, 242302 (2007).

[32] S. Afanasiev et al. (PHENIX Collaboration), Phys. Rev. C 80, 024909 (2009).

[33] Z. Xu, C. Greiner, and H. Stocker, Phys. Rev. Lett. 101, 082302 (2008).

[34] B. Schenke, S. Jeon, and C. Gale, Phys. Rev. Lett. 106, 042301 (2011).

[35] S. R. de Groot and L. G. Suttorp, Foundations of Electrodynamics (North-Holland, Amsterdam, 1972).

[36] L. Rezzolla and O. Zanotti, Relativistic Hydrodynamics (Oxford University, New York, 2013).

[37] U. Gursoy, D. Kharzeev, and K. Rajagopal, Phys. Rev. C 89, 054905 (2014).

[38] B. G. Zakharov, Phys. Lett. B 737, 262 (2014).

[39] V. Roy, S. Pu, L. Rezzolla, and D. Rischke, Phys. Lett. B 750, 45 (2015).

[40] S. Pu, V. Roy, L. Rezzolla, and D. H. Rischke, Phys. Rev. D 93, 074022 (2016).

[41] S. Pu and D. L. Yang, Phys. Rev. D 93, 054042 (2016).

[42] L. G. Pang, G. Endrodi, and H. Petersen, Phys. Rev. C 93, 044919 (2016).

[43] G. Inghirami, L. Del Zanna, A. Beraudo, M. H. Moghaddam, F. Becattini, and M. Bleicher, Eur. Phys. J. C 76, 659 (2016).

[44] A. Das, S. S. Dave, P. S. Saumia, and A. M. Srivastava, Phys. Rev. C 96, 034902 (2017).
[45] M. Greif, C. Greiner, and Z. Xu, Phys. Rev. C 96, 014903 (2017).

[46] E. Stewart and K. Tuchin, Phys. Rev. C 97, 044906 (2018).

[47] M. H. Moghaddam, B. Azadegan, A. F. Kord, and W. M. Alberico, Eur. Phys. J. C 78, 255 (2018).

[48] P. Mohanty, A. Dash, and V. Roy, Eur. Phys. J. A 55, 35 (2019).

[49] K. Tuchin, Phys. Rev. C 91, 064902 (2015).

[50] D. E. Kharzeev, J. Liao, S. A. Voloshin, and G. Wang, Prog. Part. Nucl. Phys. 88, 1 (2016).

[51] M. S. Green, J. Chem. Phys. 22, 398 (1954).

[52] R. Kubo, J. Phys. Soc. Jpn. 12, 570 (1957).

[53] Z. Xu and C. Greiner, Phys. Rev. C 71, 064901 (2005); 76, 024911 (2007).

[54] Z. Xu and C. Greiner, Phys. Rev. Lett. 100, 172301 (2008).

[55] C. Wesp, A. El, F. Reining, Z. Xu, I. Bouras, and C. Greiner, Phys. Rev. C 84, 054911 (2011).

[56] J. Uphoff, F. Senzel, O. Fochler, C. Wesp, Z. Xu, and C. Greiner, Phys. Rev. Lett. 114, 112301 (2015).

[57] M. Greif, I. Bouras, C. Greiner, and Z. Xu, Phys. Rev. D 90, 094014 (2014).

[58] Z. Chen, C. Greiner, Z. Xu, and P. Zhuang, Phys. Rev. C 100, 014906 (2019).

[59] G. S. Denicol, X. G. Huang, E. Molnar, G. M. Monteiro, H. Niemi, J. Noronha, D. H. Rischke, and Q. Wang, Phys. Rev. D 98, 076009 (2018).

[60] K. Hattori, X. G. Huang, D. H. Rischke, and D. Satow, Phys. Rev. D 96, 094009 (2017).

[61] D. Zubarev, V. Morozov, and G. Röpke, Statistical Mechanics of Nonequilibrium Processes: Relaxation and Hydrodynamic Processes (Akademie Verlag, Berlin, 1997), Vol. 2.

[62] E. A. Calzetta and B.-L. B. Hu, Nonequilibrium Quantum Field Theory (Cambridge University Press, Cambridge, England, 2008).

[63] D. J. Searles and D. J. Evans, J. Chem. Phys. 112, 9727 (2000).

[64] K. Paech and S. Pratt, Phys. Rev. C 74, 014901 (2006); 93, 059902(E) (2016).

[65] C. Cercignani and G. M. Kremer, The Relativistic Boltzmann Equation: Theory and Applications (Birkhäuser, Basel, 2002).

[66] S. R. de Groot, W. A. van Leeuwen, and Ch. G. van Weert, Relativistic Kinetic Theory: Principles and Applications (North Holland, Amsterdam, 1980).

[67] D. E. Kharzeev, L. D. McLerran, and H. J. Warringa, Nucl. Phys. A803, 227 (2008).

[68] G. S. Denicol, H. Niemi, E. Molnar, and D. H. Rischke, Phys. Rev. D 85, 114047 (2012); 91, 039902(E) (2015).

[69] G. S. Denicol, E. Molnar, H. Niemi, and D. H. Rischke, Phys. Rev. D 99, 056017 (2019). 